\title{
Epidemiologic differences between candidial and trichomonal infections as detected in cytologic smears in Taiwan
}

\author{
Pair Dong Wang ${ }^{1}$ and Ruey S Lin ${ }^{2}$ \\ ${ }^{1}$ Taipei Wanhwa District Health Center, Taipei, Taiwan; ${ }^{2}$ College of Public \\ Health, National Taiwan University, Taipei, Taiwan
}

\begin{abstract}
The epidemiologic differences between cytology-detected candidial and trichomonal infections were assessed in 15933 women attending the 12 district health centres in the Taipei area and a consecutive 1114 patients, visiting venereal disease clinics, whose smears were screened for cervical cancer between July 1991 and December 1992 in Taipei. The Pap smears were examined for the presence of specific organisms, such as trichomonas vaginalis, vaginal candida, herpes simplex virus, human papillomavirus, actinomyces, leptothrix, aspergillu, gardnerella and others. More emphasis was placed on the candidial and trichomonal infection in inflammatory Pap smears. The overall prevalence of candidial and trichomonal infections was $3.40 \%$ and $1.88 \%$, respectively. There were striking differences in the prevalence of trichomoniasis ranging from $1.74 \%$ in the district health centre population to $3.77 \%$ in the venereal disease clinic patients; however, the prevalence of candidial infection remained the same $(3.40 \%)$ in these two distinct population groups. Indices of socioeconomic status-education and personal hygiene-showed an inverse association with the prevalence of trichomoniasis but a positive correlation with candidiasis. Among participants, younger age $(<20$ years old) was independently associated with candidial $(O R=1.95)$ and trichomonal $(O R=3.87)$ infections. No sexual behavioural factors were associated with candidial infection in this study; however, having multiple sexual partners $(\mathrm{OR}=5.07)$ was associated with a significantly elevated risk of trichomoniasis, while using condoms was associated with a diminished risk $(\mathrm{OR}=0.38)$. The presence of candidiasis and trichomoniasis was highly associated with abnormal cytologic findings, particularly those indicative of inflammation. There was little evidence that findings suggestive of cervical cancer could be attributed to either candidial or trichomonal infections. These data suggest that trichomoniasis is consistent with venereal transmission of the disease, but transmission by contaminated objects cannot be ruled out because there is an increased relation between trichomoniasis infection and socioeconomic conditions and personal hygiene. Elucidation of such differences may be helpful in designing different strategies to control these infections. Furthermore, the findings can provide a good baseline of prevalence for investigating the relationship between these two pathogens and cervical dysplasia.
\end{abstract}

Keywords: candidiasis; trichomoniasis; cytologic smear; pathogen

\section{Introduction}

Infections due to vaginal candida and $T$. vaginalis are the two most common gynaecologic problems in Taiwan. ${ }^{1}$ Infections with these two pathogens are not lethal and are often considered of little significance; however, over $25 \%$ of women in Taiwan ${ }^{1}$ are chronically or intermittently infected with these common pathogens, and complications of these infections lead to infertility, ${ }^{2}$ cervical erosion and a well-known

Correspondence: Dr Pair Dong Wang, Director, Wanhwa District Health Center, No. 152, Tung-Yuan Street, Taipei, Taiwan

Accepted for publication: 27 June 1995 
source of difficulty in Pap smear diagnosis of cervical cancer. ${ }^{3,4}$ At the present time, numerous studies ${ }^{5-7}$ on these two organisms have emphasised clinical observations and therapeutic techniques. None the less, little information is available about the epidemiologic difference between candidial and trichomonal infection. Candidial infections are more common in pre-menopausal women ${ }^{7}$ in women using oral contraceptives $^{8}$ and in persons of higher socioeconomic status, ${ }^{9}$ whilst trichomonal infections are disproportionately concentrated in lower socioeconomic populations and minorities. ${ }^{10}$

Since vaginal cytologic screening is a routine procedure in Taiwan, the prevalence of candidial and trichomonal infections can be estimated. Although estimation of the rate of infection from stained smear undoubtedly underestimates the true prevalence of candidiasis and trichomoniasis, a stained smear is easily accomplished and can be a valuable by-product of cytologic screening for cervical cancer. This is contrasted with incidence studies on small groups of women who were examined by the most sensitive method, culturing for identifying Candidias and Trichomonas. ${ }^{11-13}$ In addition, because of the limited knowledge of the epidemiology of candidiasis and trichomoniasis, the present study was undertaken to examine differences in the epidemiology of these two infections, focusing on socioeconomic and sexual behavioural factors associated with cytology-detected candidial and trichomonal infections among women in Taiwan. Clarification of such differences may be useful in efforts to control these infections, and may provide a good baseline for investigating the relationship between these two common pathogens and cervical dysplasia.

\section{Materials and Methods}

From July 1991 to December 1992, a cytologic screening programme for cervical cancer was conducted in the Taipei area. Cervical smears were collected from the squamacolumnar junction of the cervix from 15933 women attending 12 district health centres and 1114 consecutive patients visiting a venereal disease clinic. Specimens were mixed on one side of a glass slide and fixed with a spray fixative. Upon arriving at the cytopathology laboratory at the Taipei pathological centre, the smears were stained by the standard Papanicolaou method. At the time of smear collection, patients were interviewed by public health nurses using a standardised questionnaire which included questions about sociodemographic and sexual behavioural factors. The cytologic diagnoses were made according to the criteria proposed by the Papanicolaou. Although they were used to screen for cervical cancer, all the smears were also examined for the presence of infections and organisms, such as candida, trichomonas, actinomyces, and for specific morphologic changes caused by such agents as human papillomavirus, herpes simplex virus and chlamydia. This study highlights the epidemiologic differences between trichomonal infection (defined as an intact organism of appropriate size and configuration with an identifiable characteristic nucleus) and candidial infection (defined as hyphae to be recognized in the stained smear).

There were too few patients with more than one type of organism to permit separate analysis and these patients were classified by the predominant organism according to the following sequence: (1) trichomonas, (2) candida, (3) other.

Although cultures have been shown to be most sensitive for identifying trichomona and candida, they are not always practical for routine use and were not used in this study because of the study aims and large number of women screened.

We analysed, using a SAS package, how the prevalence of candidial and trichomonal infections varied with sociodemographic characteristics and sexual behavioural factors. The major factors included in the analysis were the woman's age, education, personal hygiene habits, contraceptive method and number of sexual partners. We 
used the Mantel-Haenszal chi-squared test for testing for differences in the prevalence among the various factors.

In the multivariate analysis, a logistic transformation was used. First, patients with candidiasis were compared with patients with neither candidial nor trichomonal infection. Second, cases with trichomonal infection were compared with the same group. In this way, adjusted odds ratios were computed, comparing the relative odds of disease for those with the characteristic of interest (eg candidiasis or trichomoniasis) with those without the characteristic.

\section{Results}

Table 1 shows the prevalence of various infections detected on examination of inflammatory stained smears. The prevalence of candidial and trichomonal infection was $3.40 \%$ and $1.88 \%$, respectively. The total infection rate in the 17047 women was $10.37 \%$.

The prevalences of candidial and trichomonal infection at the time of study enrolment were $3.40 \%$ and $1.74 \%$, respectively, for the district health centre population, and $3.41 \%$ and $3.70 \%$, respectively, for the venereal disease clinic patients (Table 2).

Some $7.30 \%$ of the women reported having had two or more sex partners. The prevalence of candidial infection did not increase with increasing numbers of sex partner; however, there was a tendency for positive cytologic signs of trichomonal infection with greater numbers of sex partners. Another expected finding concerned the prevalence of trichomoniasis as related to age at first intercourse (Table 3). Among those who had their first sexual intercourse in their teens, the prevalence of trichomoniasis was relatively high $(4.10 \%)$ and declined steadily with greater age of

Table 1 Detection rate for various types of infection in inflammatory smears

\begin{tabular}{lcc}
\hline Type of infection & No. positive & Prevalence (\%) \\
\hline Vaginal candida & 580 & 3.40 \\
Trichomonas vaginalis & 320 & 1.88 \\
Human papillomavirus & 71 & 0.42 \\
Actinomyces & 16 & 0.09 \\
Leptothrix vaginalis & 7 & 0.04 \\
Aspergillus & 4 & 0.02 \\
Gardnerella & 3 & 0.02 \\
Other (HSV, chlamydia, bacteria etc.) & 768 & 4.51 \\
All infection & 1769 & 10.37
\end{tabular}

Note: Total women examined: 17047

Table 2 Rate of candidial and trichomonal infection, by type of health centre

\begin{tabular}{lccc}
\hline Type of health centre & $\begin{array}{c}\text { No. of } \\
\text { patients }\end{array}$ & $\begin{array}{c}\% \text { Candidial } \\
\text { infection }\end{array}$ & $\begin{array}{c}\% \text { Trichomonal } \\
\text { infection }\end{array}$ \\
\hline District health centre & 15933 & $3.40(542)$ & $1.74(278)$ \\
Venereal disease clinic & 1114 & $3.41(38)$ & $3.77(42)$ \\
Total & 17047 & $P=0.46$ & $P<0.001$ \\
& & $3.40(580)$ & $1.88(320)$ \\
\hline
\end{tabular}

Note: Figures in parentheses indicate number of women 
Table 3 Univariate analysis for sexual behavioural factors of candidiasis and trichomoniasis

\begin{tabular}{|c|c|c|c|c|c|}
\hline \multirow[t]{2}{*}{ Characteristics } & \multirow{2}{*}{$\begin{array}{l}\text { No. of } \\
\text { Patients }\end{array}$} & \multicolumn{2}{|c|}{ Candidiasis } & \multicolumn{2}{|c|}{ Trichomoniasis } \\
\hline & & $\%(N o)$. & $\begin{array}{l}\text { Odds ratio } \\
(95 \% \mathrm{CI})\end{array}$ & $\%(N o)$. & $\begin{array}{c}\text { Odds ratio } \\
(95 \% \text { CI })\end{array}$ \\
\hline \multicolumn{6}{|c|}{ Number of sexual partners } \\
\hline$<2$ & 14209 & $3.40(475)$ & 1 & $1.86(264)$ & 1 \\
\hline $2-3$ & 728 & $3.40(25)$ & $1.0(0.5-1.5)$ & $4.67(34)$ & $2.5(1.4-3.6)$ \\
\hline$>3$ & 386 & $\begin{array}{c}3.40(13) \\
P=0.27\end{array}$ & $1.0(0.5-1.6)$ & $\begin{array}{l}5.70(22) \\
P<0.001\end{array}$ & $3.0(2.6-5.3)$ \\
\hline \multicolumn{6}{|c|}{ Age at first sexual intercourse } \\
\hline $15-19$ & 1041 & $2.88(30)$ & $1.0(0.7-2.0)$ & $4.10(43)$ & $2.5(1.5-3.5)$ \\
\hline $20-29$ & 14024 & $3.45(484)$ & $1.2(1.0-1.9)$ & $1.74(244)$ & $1.1(0.8-1.6)$ \\
\hline$>30$ & 1174 & $2.81(33)$ & & $1.62(19)$ & 1 \\
\hline & & $P=0.12$ & & $P<0.001$ & \\
\hline
\end{tabular}

Note: Because of missing data, numbers available of each variable were often less than the total women examined

first intercourse. In contrast, less variation in candidiasis prevalence was observed when stratified by age of first sexual intercourse.

The prevalence of both candidial and trichomonal infections was greatest in adolescents and declined with age, especially in those over 60 (Table 4).

There was no clear relation between ethnicity and candidiasis; however, trichomoniasis was most common in the aboriginal group $(3.61 \%)$, compared with $1.97 \%$ in mainlanders and $1.87 \%$ in Taiwanese.

The prevalence of candidiasis had a significant association with educational status. Women with a college or higher education had the highest prevalence of candidiasis $(3.70 \%)$ as compared with illiterate women $(2.70 \%)$. In contrast, for trichomonal infection, the association with educational level was reversed. Trichomoniasis was most common in illiterate women $(2.72 \%)$ and lowest $(0.94 \%)$ in women with a college or higher education.

There was no clear association between genital hygiene and candidiasis prevalence; however, trichomoniasis was most common in women reporting that they never or seldom washed the genital area after sexual intercourse $(2.61 \%)$ compared with $1.98 \%$ in women who reported that they usually washed (Table 4).

In the total population studied, candidial infections were found in $3.14 \%$ of the women who used oral contraceptives and in $3.72 \%$ of those who did not. In contrast, only $0.94 \%$ of the women using condoms harbored trichomonas whereas $2.25 \%$ of those who did not use condoms had trichomonal infections.

To further evaluate the relative importance of variables found to be associated with candidial or trichomonal infections, we performed multivariate logistic regression analyses (Table 5). To determine which factors were independently associated with likelihood of candidiasis or trichomoniasis, we created models incorporating age, multiple sexual partners, educational level, genital hygiene and contraceptive method.

In the model, being under 20 years of age, having sex with more than one partner, reporting poor genital hygiene, and not using condoms were each independently associated with greater likelihood of trichomoniasis in comparison with women without candidial or trichomonal infection (Table 5). In a similar analysis, only age differentiated females with candidial infection from those without either candidiasis or trichomoniasis. 
Table 4 Univariate analysis for sociodemographic characteristics of candidiasis and trichomoniasis, Taiwan 1991-92

\begin{tabular}{|c|c|c|c|c|c|}
\hline \multirow[t]{2}{*}{ Characteristics } & \multirow{2}{*}{$\begin{array}{l}\text { No. of } \\
\text { patients }\end{array}$} & \multicolumn{2}{|c|}{ Candidiasis } & \multicolumn{2}{|c|}{ Trichomoniasis } \\
\hline & & $\%(N o)$, & $\begin{array}{l}\text { Odds ratio } \\
(95 \% \text { CI })\end{array}$ & $\%(N o)$. & $\begin{array}{l}\text { Odds ratio } \\
(95 \% \mathrm{Cl})\end{array}$ \\
\hline \multicolumn{6}{|l|}{ Age (years): } \\
\hline $15-19$ & 76 & $6.58(5)$ & 1 & $7.89(6)$ & 1 \\
\hline $20-29$ & 3795 & $3.53(134)$ & $0.5(0.3-0.5)$ & $1.77(67)$ & $0.2(0.1-0.9)$ \\
\hline $30-39$ & 8900 & $3.75(334)$ & $0.6(0.3-0.9)$ & $1.71(152)$ & $0.2(0.1-0.8)$ \\
\hline $40-49$ & 2914 & $3.16(92)$ & $0.5(0.2-0.9)$ & $2.57(75)$ & $0.3(0.1-0.9)$ \\
\hline $50-59$ & 963 & $1.04(10)$ & $0.2(0.1-1.8)$ & $1.87(18)$ & $0.2(0.1-1.4)$ \\
\hline $60+$ & 396 & $\begin{array}{r}1.26(5) \\
P<0.001\end{array}$ & $0.2(0.1-2.0)$ & $\begin{array}{r}0.51(2) \\
P<0001\end{array}$ & $0.1(0.1-2.5)$ \\
\hline \multicolumn{6}{|l|}{ Ethnic group: } \\
\hline Taiwanese & 13933 & $3.25(453)$ & 1 & $1.87(261)$ & 1 \\
\hline Mainlander & 2692 & $4.29(115)$ & $1.3(1.0-1.9)$ & $1.97(53)$ & $1.1(0.7-1.8)$ \\
\hline Aboriginal & 166 & $\begin{array}{c}3.01(5) \\
P=0.07\end{array}$ & $0.9(0.6-2.0)$ & $\begin{array}{c}3.61(6) \\
P<0.05\end{array}$ & $1.9(1.1-5.9)$ \\
\hline \multicolumn{6}{|l|}{ Education level: } \\
\hline Illiterate & 515 & $2.72(14)$ & $0.7(0.4-2.1)$ & $2.72(14)$ & $2.9(1.5-4.6)$ \\
\hline Primary & 3302 & $2.76(91)$ & $0.7(0.6-1.6)$ & $2.51(83)$ & $2.7(1.4-3.3)$ \\
\hline Junior high & 2810 & $3.20(90)$ & $0.9(0.7-1.6)$ & $2.06(58)$ & $2.2(1.3-3.2)$ \\
\hline Senior high & 6815 & $3.68(251)$ & $1.0(0.8-1.5)$ & $1.95(133)$ & $2.1(1.3-2.9)$ \\
\hline \multirow[t]{2}{*}{ College and over } & 3394 & $3.71(126)$ & 1 & $0.94(32)$ & 1 \\
\hline & & $P=0.08$ & & $P<0.05$ & \\
\hline \multicolumn{6}{|c|}{ Genital hygiene (washing the genital area after sexual intercourse): } \\
\hline usually & 8759 & $3.23(283)$ & 1 & $1.98(171)$ & 1 \\
\hline \multirow[t]{2}{*}{ never or seldom } & 5705 & $3.23(184)$ & $1.0(0.8-1.5)$ & $2.61(149)$ & $1.3(1.1-1.7)$ \\
\hline & & $P=0.36$ & & $P<0.05$ & \\
\hline \multicolumn{6}{|l|}{ Contraceptive method: } \\
\hline none & 3068 & $2.41(74)$ & 1 & $1.99(61)$ & 1 \\
\hline condom use & 2666 & $3.90(104)$ & $1.6(1.2-2.2)$ & $0.94(25)$ & $0.5(0.2-0.8)$ \\
\hline oral contraceptives & 1373 & $3.13(43)$ & $1.3(0.9-1.9)$ & $2.40(33)$ & $1.2(0.9-1.9)$ \\
\hline \multirow[t]{2}{*}{ other } & 8584 & $4.18(359)$ & $1.7(1.2-2.1)$ & $2.34(201)$ & $1.2(1.0-1.8)$ \\
\hline & & $P=0.17$ & & $P<0.01$ & \\
\hline
\end{tabular}

Note: Because of missing data, numbers available of each variable were often less than the total women examined

Table 5 Adjusted odds ratio for candidial or trichomonal infection among women in Taiwan, 1991-92

\begin{tabular}{|c|c|c|c|c|}
\hline \multirow[t]{2}{*}{ Factor } & \multicolumn{2}{|c|}{ Candidiasis } & \multicolumn{2}{|c|}{ Trichomoniasis } \\
\hline & Odds ratio & $95 \% C I$ & Odds ratio & $95 \% \mathrm{Cl}$ \\
\hline Age $<20$ years & 1.95 & $1.03-2.46^{*}$ & 3.87 & $2.29-8.35^{* *}$ \\
\hline$>1$ sexual partner & 1.04 & $0.51-1.98$ & 5.07 & $3.97-10.05^{* *}$ \\
\hline Education below junior high & 0.91 & $0.53-1.37$ & 1.86 & $1.02-2.21^{*}$ \\
\hline Poor genital hygiene & 1.01 & $0.69-1.41$ & 1.46 & $1.01-2.70^{*}$ \\
\hline Condon use & 0.96 & $0.96-1.39$ & 0.38 & $0.26-0.64^{*}$ \\
\hline
\end{tabular}

Note: ${ }^{*} P<0.05 ; * * P<0.01$ 
The presence of candida and trichomonas was found to be highly associated with abnormal cytologic findings (Table 6); $28.41 \%$ and $16.59 \%$ of the women with cytologic changes classified as inflammatory had demonstrable candidiasis and trichomoniasis, respectively. Only $1.35 \%$ and $2.03 \%$ of those with suggestive evidence suspicious of neoplasia showed the presence of candidiasis and trichomoniasis, respectively.

\section{Discussion}

As can be seen from Table 1 , it is interesting that the prevalence of candidiasis in the district health centre population and venereal disease clinic patients was approximately the same, while trichomoniasis was determined by cytologic signs in $1.74 \%$ of the district health centre women and $3.77 \%$ of venereal disease clinic patients. Our finding of a higher prevalence of trichomoniasis in venereal disease clinic patients confirms the findings of a number of other studies. ${ }^{14,15}$ Certainly, the venereal disease clinic patients with promiscuous heterosexual contacts had a high prevalence of infection, whereas the district health centre women were probably married and had, for the most part, had no extramarital sexual contacts. In addition, evaluation of this large cohort of women demonstrated several novel aspects of the epidemiology of trichomonal infection among women in Taiwan. Among participants, trichomonal infection was related to several aspects of sexual activity, such as increased number of sexual partners and a younger age at first sexual intercourse, and was inversely related to use of condoms. Our data suggest that sexual transmission plays an important role in the acquisition of trichomonas. These epidemiologic features were not present among women with candidial infection. Thus, the characteristic of candidial infection in the study can be interpreted as suggesting that candidiasis is not officially classified as a sexually transmitted disease, and is less clearly associated with behavioural factors such as multiple sex partners, ${ }^{8,9}$

Young age was associated with higher infection rates for both organisms. Younger females tend to have larger zones of cervical ectropion and therefore may have a larger number of potential target cells for candidial and trichomonal infection than older patients. Another possibility is that, in older persons, immunity derived from previous infections may reduce the subsequent likelihood of detectable candidial infection or of trichomonal infection by organisms of the same serovar.

It is interesting to observe the difference in prevalence of both agents in the preand postmenopausal women. Although these represent two different age-groups, and therefore a number of variables are involved, it seems likely that menstruating women

Table 6 Candidial and trichomonal infections and Pap smear results

\begin{tabular}{|c|c|c|c|c|c|c|}
\hline \multicolumn{2}{|c|}{ Pap smear classification } & \multirow[t]{2}{*}{ No. } & \multicolumn{2}{|c|}{$\begin{array}{l}\text { Positive smears } \\
\text { for candida }\end{array}$} & \multicolumn{2}{|c|}{$\begin{array}{l}\text { Positive smears } \\
\text { for trichomonas }\end{array}$} \\
\hline & & & No. & $\%$ & No. & $\%$ \\
\hline Class I & Normal cytology & 14614 & 35 & 0.24 & & \\
\hline Class If & Compatible with inflammation & 1911 & 543 & 28.41 & 317 & 16.59 \\
\hline Class III & Suspicious of neoplasia & 148 & 2 & 1.35 & 3 & 2.03 \\
\hline Class IV & Compatible with neoplasia & 22 & & & & \\
\hline Class V & Definite malignant changes & 13 & & & & \\
\hline Inadequate & & 348 & & & & \\
\hline Total & & 17056 & 580 & 3.40 & 320 & 1.88 \\
\hline
\end{tabular}


provide a more satisfactory living environment for these organisms than do postmenopausal women.

Finally, while infection with either candida or trichomonas was more common in younger women, the particularly strong association of trichomonal infection with young age is noteworthy. Compared with candidiasis, the likelihood of trichomonal infection in teenagers was fourfold that for women aged 20 or older. This observation is also consistent with a stronger influence of immunity derived from prior infection for trichomoniasis than for candidiasis.

The prevalence of candidiasis had a positive association with educational status. Women with a college or higher education had the highest prevalence $(3.70 \%)$ as compared with illiterate women $(2.9 \%)$. In contrast, highest frequencies of trichomonal infection were found in illiterate women. It is probably true that educated women may maintain better genital hygiene and that poor genital hygiene facilitates the transmission of trichomoniasis.

Of the contraceptive methods analysed, the influence of oral contraceptives on vaginal candidiasis has not yet been clearly defined. Both Walsh, ${ }^{16}$ using cultures, and Wied, ${ }^{17}$ using stained smears, reported that candidial infections were significantly increased in women taking oral contraceptives. Our data and other studies ${ }^{17-19}$ failed to confirm these findings. The association between use of condoms and decreased trichomonal but not candidial infection was also notable. Our findings support the hypothesis that condoms may protect against the acquisition of trichomonas.

The difference between the sociodemographics and between sexual behaviours associated with candidiasis and trichomoniasis observed in this study suggest that although non-venereal acquisition of trichomonas has been reported, ${ }^{20,21}$ trichomoniasis is mainly transmitted by sexual contact. Thus, from a public health perspective, our findings suggest that avoiding sexual promiscuity and using barrier contraception might contribute to prevention of sexual transmission of trichomonas. In contrast, our data suggest that candidiasis is not related to sexual behaviour, and that non-sexual routes or exogenous reinfection exist for candidial transmission. These data are also consistent with observation of other investigators. ${ }^{22}$ Understanding these findings may help to prevent candidial infection.

The presence of candidiasis and trichomoniasis was highly associated with abnormal cytologic findings, as has been reported by other investigators, ${ }^{3,23}$ particularly those indicative of inflammation. There was little evidence that findings suggestive of neoplasia could be attributed to either candidiasis or trichomoniasis. This result was also supported by Guijon's study. ${ }^{24}$ In addition, by treating all patients with inflammatory atypia medically, improvement of the smear following medication should be a useful method of differentiating changes produced by candida or trichomonas from premalignant lesions of the cervix. ${ }^{25}$ If the smears demonstrate persistent inflammatory atypia, then recommendations should be made for the patient to undergo colposcopic evaluation to rule out intraepithelial neoplasia. ${ }^{26}$

\section{Acknowledgements}

The authors thank all the cytotechnicians involved in this study, 16 public health nurses for their interview, and Professor Yuh Y Pan for computer programming.

\section{References}

1 Wei RD. Leukorrhea and Vaginitis, Clinical Gynecology. Ma-tzu, Publishers: Taipei, Taiwan, 1983, pp 197-203.

2 Tuttle JP, Halbrook TW, Derrick FC. Interference of human spermatozol mobility by trichomonas vaginalis. $J$ Urol 1977; 118: 1024-1025. 
3 Simeckova M, Lonser E, Nichols E, Rubinstein I. Chronic Trichomoniasis and cervical cancer. Obstet Gynecol 1962; 20: 410-416.

4 Weatherby M. The incidence and significance of Trichomonas infestations. Southern Med J 1962; 55: 1044-1049.

5 Pickhardt WL, Breen JL. Identification and therapy for vaginal candidiasis. Am J Obstet Gynecol 1957; 74: 42-47.

6 O'Brian JR. Clinical trials with agents currently used in the management of vaginitis. Canad Med Ass J 1964; 90: 1206-1210.

7 Gray LA, Barnes ML. Vaginitis in women, diagnosis and treatment. Am J Obstet Gynecol 1965; 272: 647-656.

8 Yaffee HS, Grots RI. Moniliasis due to norethynodril with mestranol. New Engl J Med 1965; 272: 647-656.

9 Davis BA. Vaginal moniliasis in private practice. Obstet Gynecol 1969; 34: 40-45.

10 Trussel RE. Trichomonas Vaginalis and Trichomoniasis. Charles C. Thomas: Springfield, IL, 1947.

11 McCann JS. Comparison of direct microscopy and culture in the diagnosis of trichomoniasis. Br J Vener Dis 1974; 50: 450-458.

12 Spence MR, Hollander DH, Smith $\mathrm{H}$ et al. The clinical and laboratory diagnosis of Trichomonas vaginalis infection. Sex Trans Dis 1980; 7: 168-173.

13 Kean BH, Day E. Trichomonas vaginalis infection. An evaluation of three diagnostic techniques with data on incidence. Am J Obstet Gynecol 1954; 68: 1510-1518.

14 Krieger JN, Tam MR, Stevens CE, Nielsen IO et al. Diagnosis of Trichomoniasis. JAMA 1988; 259: 1223-1227.

15 Mclellan R, Spence MR, Smith JL. The clinical diagnosis of trichomoniasis. Obstet Gynecol 1982; 60: 30-34.

16 Walsh $\mathrm{H}$, Hildebrandt RJ, Prystowsky $\mathrm{H}$. Oral progestational agents as a cause of candida vaginitis. Am J Obstet Gynecol 1968; 27: 327-334.

17 Wied GL, Davis ME, Frank R et al. Statistical evaluation of the effect of hormonal contraceptives on the cytologic smear pattern. Obstet Gynecol 1966; 27: 327-334.

18 Spellacy WN, Zais N, Buhi WC et al. Vaginal yeast growth and contraceptive practices. Obstet Gynecol 1971; 38: 343-349.

19 Lapan B. Is the 'Pill' a cause of vaginal candidiasis? Culture study. NY State J Med 1970; 70: $949-951$.

20 Catteral RD, Nicol CS. Is trichomonal infestation a venereal disease? $B M J 1960$; 1: $117-1180$.

21 Whittington MJ. Epidemiology of infection with Trichomonas vaginalis in the light of improved diagnostic methods. $B r J$ Vener Dis 1957; 33: 80-85.

22 Seelig MS. The role of antibiotics in the pathogenesis of Candida infections. Am $J$ Med 1966; 40: $887-893$.

23 Ayer JE. Role of the halo cells in cervical carcinogenesis. Obstet Gynecol 1960; 15: $481-487$.

24 Guijon FB, Paraskevas M, Burnham R. The association of sexually transmitted diseases with cervical intraepithelial neoplasia: a case-control study. Obstet Gynecol 1985; 68: $251-257$.

25 Weatherby $M$. The incidence and significance of Trichomonas infestations. $B M J 1962 ; 55$ : 1044-1049.

26 Noumoff JS. Atypia in cervical cytology as a risk factor for intraepithelial neoplasia. Am J Obstet Gynecol 1987; 156: 628-631. 